\title{
Immunogeno: Protective mechanism for Rift Valley fever in the Democratic Republic of Congo
}

\author{
Author: \\ Georges Tshilenge ${ }^{1}$ \\ Affiliation: \\ ${ }^{1}$ Central Veterinary \\ Laboratory, Kinshasa 1, \\ Democratic Republic of \\ Congo \\ Correspondence to: \\ Georges Tshilenge \\ Email: \\ george.tshilenge@sacids.org \\ Postal address: \\ PO Box 8842, Kinshasa 1, \\ Democratic Republic of \\ Congo \\ How to cite this poster: \\ Tshilenge, G., 2012, \\ 'Immunogeno: Protective \\ mechanism for Rift Valley \\ fever in the Democratic \\ Republic of Congo', \\ Onderstepoort Journal of \\ Veterinary Research 79(2), \\ Art. \#483, 1 page. http:// \\ dx.doi.org/10.4102/ojvr. \\ v79i2.483 \\ Note: \\ Proceedings of the \\ Conference of the Southern \\ African Centre for Infectious \\ Disease Surveillance 'One \\ Health' held at the National \\ Institute for Communicable \\ Diseases, Johannesburg, July \\ 2011.
}

(C) 2012. The Authors.
Rift Valley fever (RVF) is an acute, fever causing viral disease that affects domestic animals and humans.

In Democratic Republic of Congo (DRC), this pathology is not well documented. No epidemic of the RVF has not been reported but sera samples collected in six provinces surveyed from 2005 to 2006 revealed $14 \%$ of apparent prevalence and, high apparent prevalence $(20 \%)$ of antibodies against RVF virus was reported in Katanga Province during the same survey; this serological evidence was associated with abortions cases in Cattle (Mulumba et al. 2009). Livestock immunisation is important for control of Rift Valley fever virus (RVFV) epidemics; however immunisation of susceptible domestic animals in endemic countries does not protect animals against the clinical disease but prevents the propagation of virus to human population through reduction of the amplification degree in host animals. The humoral immunity is sufficient for protection for animals as well as for humans. The infection caused by RVFV leads to neutralisation of the immunity of the animal (Barnard 1979).

Various immunological studies have been made on the characterisation of immune response during RVFV epidemics but, until now several studies have been concentrated on the response of the innate immune particularly based on signal interferon system than the response of the adaptive immune and cell mediated humoral immune. The available information on the immune response related to RVFV does not seem to provide enough information on various mechanisms of the response immune system.

The aim of the study is based on mechanism of immune response system including protective effect of immunisation against RVFV. In addition, epidemiological and molecular studies will be assessed. As a matter of fact, following studies will be conducted:

- evaluation of the immunological protection against Rift Valley fever in vaccinated and nonvaccinated cattle using IgG and IgM ELISAs in Katanga Province

- assessment of cellular response to Rift Valley fever disease in vaccinated and naturally infected cattle

- molecular characterisation of RVFV strains circulating in vaccinated and non vaccinated cattle

- assessment of protective effect related to vaccinal strains in cattle, using a longitudinal survey.

The studies will be carried out Northern Katanga Province within two areas, one with historyof circulation of RVFV and other without history RVFV circulation.

Whole blood, spleen, liver, lymph node will be collected as target tissues from cattle carcasses. In addition, goats and sheeps samples will be collected alongside from each area in order to clarify the disease situation. Serological tests based on the detection of Ig M and Ig G will be used. DIVA tests, LAMP, and IHC techniques will be used. Within previously vaccinated areas in the above mentioned areas and those that are not vaccinated, the collected samples will be analysed using RT-PCR/RT-LAMP.

In vitro experimental studies systems will be carried out using animal PMBCs that will be infected with wild type of RVF virus as well as with vaccinal strains, such as clones 13 and MP12 to characterise various cell types such as CD4 T cells, CD8 T cells, B-cells, NK cells and, macrophages will be studied with regard to activation and apoptosis signals on various post - infection days, using flow cytometry. A pool of animals will be vaccinated with the Clone 13 and another with the MP12 to determine the traceability. The monitoring of the immune response will be done through the measurement of immunoglobulin G (Ig G) and immunoglobulin M (Ig M). RT-PCR, spectrophotometer or Facs methods will be used for the dosage of cells T CD4 + and Cell T CD8+. 